\title{
COVERS OF ALGEBRAIC VARIETIES VI. ANGLO-AMERICAN COVERS AND $(1,3)$-POLARIZED ABELIAN SURFACES
}

\author{
Gianfranco Casnati
}

\begin{abstract}
In the present paper we describe a class of Gorenstein, finite and flat morphism $\varrho: X \rightarrow Y$ of degree 6 of algebraic varieties, called Anglo-American covers. We prove a general Bertini theorem for them and we give some evidence that the cover $\varrho: A \rightarrow \mathbb{P}_{k}^{2}$ associated general $(1,3)$-polarized abelian surface is Anglo-American.
\end{abstract}

\section{Introduction and notations}

In the paper [7], T. Ekedahl and the author proved a general factorization theorem for covers of degree $d \geq 3$ of algebraic varieties satisfying a technical condition, namely Gorenstein covers, e.g. surjective and finite morphisms of degree $d$ between smooth varieties over the complex field $\mathbb{C}$.

Such a result has been used in order to give a complete characterization of Gorenstein covers when their degree is either $d=3,4$ (see [7]) or $d=5$ (see [3]).

In the last case, when $d=5$, we defined a projective bundle $\bar{\pi}: \overline{\mathbb{P}} \rightarrow Y$ of rank $n+d-2$, a subbundle $\mathbb{P} \subseteq \overline{\mathbb{P}}$ of rank $d-2$ and a suitable subscheme $V \subseteq \overline{\mathbb{P}}$ of relative dimension $n$ which is fibrewise a suitable del Pezzo variety of degree $d$. If, fibrewise over $Y, X:=V \cap \mathbb{P}$ is of dimension 0 , then $\varrho=\bar{\pi}_{\mid X}: X \rightarrow Y$ is a cover. So it is natural to ask if it is possible to construct interesting classes of covers of degree $d \geq 6$ via this method.

In [5] we dealed with a first class of covers of degree 6 , corresponding fibrewise to the Segre embedding $\mathbb{P}_{k}^{2} \times \mathbb{P}_{k}^{2} \subseteq \mathbb{P}_{k}^{8}$ and called Scandinavian.

In [6] we described a method for producing covers of degrees 8 and 9 , respectively related to the 2 -uple embedding $\mathbb{P}_{k}^{3} \subseteq \mathbb{P}_{k}^{9}$ and to the 3 -uple embedding $\mathbb{P}_{k}^{2} \subseteq \mathbb{P}_{k}^{9}$. We also suggested the existence of a method for constructing covers of degree 6 corresponding fibrewise to the Segre embedding $\mathbb{P}_{k}^{1} \times \mathbb{P}_{k}^{1} \times \mathbb{P}_{k}^{1} \subseteq \mathbb{P}_{k}^{7}$ and called Anglo-American. We also stated that such covers are always trivial, in the sense that they factorize through a sequence of finite morphisms of degree

Received April 17, 2008; Revised December 6, 2010.

2010 Mathematics Subject Classification. 14E20, 14E22.

Key words and phrases. cover of degree d, Anglo-American cover. 
2 and 3. Unfortunately the proof contains a silly mistake and the factorization statement is actually false (see Example 4.1).

The first motivation of the present paper is to describe such class of covers (see Section 2) and to prove a general Bertini theorem for them (see Section 3) similar to the ones proved in $[7,3,5]$ and $[6]$.

Using such a result, we give in Section 4 some examples. In particular, we construct a non-factorizing Anglo-American cover $\varrho: X \rightarrow \mathbb{P}_{k}^{2}$ where $X$ is an integral smooth surface and we check the non-surprising fact that the classes of Scandinavian and Anglo-American covers are not contained one into the other. We also construct a cover which is neither Anglo-American nor Scandinavian. Notice that these covers cannot be distinguished from their fibres as pointed out in Sections $6.1,6.2,6.3$ of [8]: it is necessary to look at them globally.

Perhaps the more interesting example of cover of degree 6 is related to a general $(1,3)$-polarized abelian surface $A$. In this case the polarization induces naturally a cover $\varrho: A \rightarrow \mathbb{P}_{k}^{2}$ of degree 6 . Except for its branch locus, that has been studied in [15], not very much is known about such a $\varrho$. From this viewpoint, the unique known result regards the structure of the sheaf $\varrho_{*} \mathcal{O}_{A}$ (see [4]), which forces $\varrho$ to be non-Scandinavian as proved in [5]. Thus, it is quite natural to ask if it is Anglo-American. As explained in Section 5 there is some evidence that this could be true: unfortunately the existence theorem cannot be applied in this case, hence we are unable to prove such a result for $\varrho$.

Notations. From now on $k$ will denote an algebraically closed field of characteristic $p \geq 0$. A scheme $X$ is a Noetherian scheme. A scheme $X$ over $k$ is a scheme which is separated and of finite type over $k$.

Let $Y$ be an irreducible scheme, $X$ another scheme. A cover $\varrho: X \rightarrow Y$ is a flat and finite morphism. Its degree $d$ is, by definition, the rank of the locally free $\mathcal{O}_{Y}$-sheaf $\varrho_{*} \mathcal{O}_{X}$.

If $\varrho: X \rightarrow Y$ is a cover of degree $d$, then we have the trace map $\varrho^{\#}: \mathcal{O}_{Y} \rightarrow$ $\varrho_{*} \mathcal{O}_{X}$. The sheaf coker $\varrho^{\#}$ is a locally free $\mathcal{O}_{Y}$-sheaf of rank $d-1$ called the

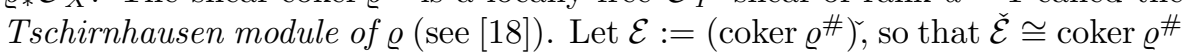
(for each sheaf $\mathcal{F}$ on $Y$ we denote by $\check{\mathcal{F}}$ its dual, i.e., $\check{\mathcal{F}}:=\mathcal{H}_{\text {or }}$ O $_{Y}\left(\mathcal{F}, \mathcal{O}_{Y}\right)$ ). We have an exact sequence $0 \rightarrow \mathcal{O}_{Y} \stackrel{\varrho^{\#}}{\longrightarrow} \varrho_{*} \mathcal{O}_{X} \rightarrow \check{\mathcal{E}} \rightarrow 0$. If $Y$ is defined over a field $k$ whose characteristic $p$ does not divide $d$, then the sequence above splits, hence

$$
\varrho_{*} \mathcal{O}_{X} \cong \mathcal{O}_{Y} \oplus \check{\mathcal{E}}
$$

(generalize the proof of Lemma 2.2 in [18]).

A cover $\varrho: X \rightarrow Y$ is called Gorenstein if the scheme-theoretic fibre $\varrho^{-1}(y)$ is a locally Gorenstein scheme over $k(y)$ for every $y \in Y$ : this is equivalent to the invertibility of the relative dualizing sheaf $\omega_{X \mid Y}$. If $Y$ is Gorenstein, then $\varrho$ is Gorenstein if and only if so is $X$. If $\varrho$ is Gorenstein relative duality yields a natural exact sequence $0 \rightarrow \mathcal{E} \rightarrow \varrho_{*} \omega_{X \mid Y} \rightarrow \mathcal{O}_{Y} \rightarrow 0$. 
For all the other notations and results we always refer to [12].

\section{The scheme of totally reducible tensors}

Let $T$ be a scheme and let $\mathcal{U}_{i}, 1 \leq i \leq 3$ be three locally free $\mathcal{O}_{T}$-sheaves of rank 2. Consider the Segre embedding $S: \mathbb{P}\left(\mathcal{U}_{1}\right) \times_{T} \mathbb{P}\left(\mathcal{U}_{2}\right) \times_{T} \mathbb{P}\left(\mathcal{U}_{3}\right) \rightarrow \mathbb{P}(\mathcal{U})$ where $\mathcal{U}:=\mathcal{U}_{1} \otimes \mathcal{U}_{2} \otimes \mathcal{U}_{3}$.

Definition 2.1. The locus $\mathcal{D}_{t r}$ of totally reducible tensors is the image inside $\mathbb{P}(\mathcal{U})$ of $S$.

When $T=\operatorname{spec}(k)$ we can consider homogeneous coordinates $u_{0}^{(i)}, u_{1}^{(i)}$ in $\mathbb{P}_{k}^{1}$, $i=1,2,3$ : thus we have induced coordinates $u_{i, j, h}, 0 \leq i, j, h \leq 1$ in $\mathbb{P}_{k}^{7}$ and $S$ is given by $u_{i, j, h}=u_{i}^{(1)} u_{j}^{(2)} u_{h}^{(3)}, 0 \leq i, j, h \leq 1$.

Lemma 2.2. $\mathcal{D}_{t r} \subseteq \mathbb{P}_{k}^{7}$ is an arithmetically Gorenstein subscheme of degree 6 with minimal free resolution of the form

$$
\begin{aligned}
0 \longrightarrow \mathcal{O}_{\mathbb{P}_{k}^{7}}(-6) & \longrightarrow \mathcal{O}_{\mathbb{P}_{k}^{7}}(-4)^{\oplus 9} \longrightarrow \mathcal{O}_{\mathbb{P}_{k}^{7}}(-3)^{\oplus 16} \longrightarrow \\
& \longrightarrow \mathcal{O}_{\mathbb{P}_{k}^{7}}(-2)^{\oplus 9} \longrightarrow \mathcal{O}_{\mathbb{P}_{k}^{7}} \longrightarrow \mathcal{O}_{\mathcal{D}_{t r}} \longrightarrow 0
\end{aligned}
$$

The homogeneous ideal of $\mathcal{D}_{\text {tr }}$ in $k\left[u_{i, j, h}\right]_{0 \leq i, j, h \leq 1}$ is generated by the determinants of all the faces of the cubic array $\left(u_{i, j, h}\right)_{0 \leq i, j, h \leq 1}$.

Proof. The sheaf $\mathcal{O}_{\mathbb{P}_{k}^{7}}(1)$ restricts to $\mathcal{D}_{t r}$ to the tensor product of the pullbacks of $\mathcal{O}_{\mathbb{P}_{k}^{1}}(1)$ from the three copies of $\mathbb{P}_{k}^{1}$. It follows that $\omega_{\mathcal{D}_{t r}} \cong \mathcal{O}_{\mathcal{D}_{t r}}(-2)$. Moreover, Künneth formulas also give $H^{i}\left(\mathcal{D}_{t r}, \mathcal{O}_{\mathcal{D}_{t r}}(n)\right)=0$ when $i=1,2$, whence it follows that $\mathcal{D}_{t r}$ is arithmetically Gorenstein.

Let $\mathcal{D}$ be the image of the Segre embedding $\mathbb{P}_{k}^{1} \times \mathbb{P}_{k}^{3} \subseteq \mathbb{P}_{k}^{7}$. Then $\mathcal{D}$ is the locus of $2 \times 4$ matrices of rank at most 1: we denote by $p$ and $q$ the projections of $\mathcal{D}$ on the first and second factor. Obviously we have inclusions $\mathcal{D}_{t r} \subseteq \mathcal{D} \subseteq \mathbb{P}_{k}^{7}$ and the following exact sequence of $\mathcal{O}_{\mathcal{D}}$-sheaves

$$
0 \longrightarrow q^{*} \mathcal{O}_{\mathbb{P}_{k}^{3}}(-2) \longrightarrow \mathcal{O}_{\mathcal{D}} \longrightarrow \mathcal{O}_{\mathcal{D}_{t r}} \longrightarrow 0 \text {. }
$$

Notice that $q^{*} \mathcal{O}_{\mathbb{P}_{k}^{3}}(-2) \cong p^{*} \mathcal{O}_{\mathbb{P}_{k}^{1}}(2) \otimes \mathcal{O}_{\mathbb{P}_{k}^{7}}(-2)_{\mid \mathcal{D}}$. A minimal free resolution of $p^{*} \mathcal{O}_{\mathbb{P}_{k}^{1}}(b) \otimes \mathcal{O}_{\mathbb{P}_{k}^{7}}^{(}(a)_{\mid \mathcal{D}}$ can be computed in terms of the generalized EagonNorthcott complex $\mathcal{C}^{b} \otimes \mathcal{O}_{\mathbb{P}_{k}^{7}}(a)$ for each $b \geq-1$ (see Section 1 of [20] and the references therein). Thus a mapping cone $\mathcal{C}^{2} \otimes \mathcal{O}_{\mathbb{P}_{k}^{7}}(-2) \rightarrow \mathcal{C}^{0}$ yields the sequence $(2.2 .1)$.

We finally notice that the sequence (2.2.1) implies that the homogeneous ideal of $\mathcal{D}_{t r}$ in $k\left[u_{i, j, h}\right]_{0<i, j, h<1}$ is generated by nine quadratic forms. The nine quadratic forms

$$
\begin{array}{lll}
u_{0,0,0} u_{1,0,1}-u_{1,0,0} u_{0,0,1}, & u_{1,1,0} u_{0,1,1}-u_{1,1,1} u_{0,1,0}, & u_{0,0,0} u_{1,1,0}-u_{1,0,0} u_{0,1,0} \\
u_{0,0,1} u_{1,1,1}-u_{1,0,1} u_{0,1,1}, & u_{1,1,1} u_{0,0,0}-u_{1,0,0} u_{0,1,1}, & u_{1,0,1} u_{0,1,0}-u_{1,1,0} u_{0,0,1} \\
u_{0,0,0} u_{0,1,1}-u_{0,0,1} u_{0,1,0}, & u_{1,1,0} u_{1,0,1}-u_{1,0,0} u_{1,1,1}, & u_{0,0,1} u_{1,1,0}-u_{1,0,0} u_{0,1,1}
\end{array}
$$


are linearly independent and vanish on $\mathcal{D}_{t r}$, thus they can be chosen as generators of such an ideal. Moreover, it is easy to check that the above forms are nine of the twelve determinants obtained by the faces of the cubic array $\left(u_{i, j, h}\right)_{0 \leq i, j, h \leq 1}$ and that the remaining three determinants depend linearly on the above ones.

Let $C \mathcal{D}_{t r} \subseteq \mathbb{V}(\mathcal{U})$ be the cone over $\mathcal{D}_{t r}$.

Definition 2.3. If $\varphi \in H^{0}(T, \check{\mathcal{U}})$ we define the locus of total reducibility of $\varphi$ as $D_{t r}(\varphi):=\varphi^{-1}\left(C \mathcal{D}_{t r}\right) \subseteq T$.

Remark 2.4. If $\varphi$ is defined locally around $t \in T$ by the tensor $\varphi_{i, j, h}$, then the sheaf of ideals of $D_{t r}(\varphi)$ is generated by the determinants of all the faces of the cubic array $\left(\varphi_{i, j, h}\right)_{0 \leq i, j, h \leq 1}$ locally around $t$.

We can now prove the following:

Proposition 2.5. Let $k:=\mathbb{C}, T$ be an integral, smooth projective scheme over $k$ with $\operatorname{dim}(T) \leq 7$ and $\mathcal{U}_{i}, 1 \leq i \leq 3$ be three locally free $\mathcal{O}_{Z}$-sheaves of ranks 2 and set $\mathcal{U}:=\mathcal{U}_{1} \otimes \mathcal{U}_{2} \otimes \mathcal{U}_{3}$.

If $\check{\mathcal{U}}$ is globally generated, for each general $\varphi \in H^{0}(T, \check{\mathcal{U}})$ the scheme $D_{t r}(\varphi)$ is smooth.

Proof. Since $T$ is smooth the scheme $C \mathcal{D}_{t r} \subseteq \mathbb{V}(\mathcal{U})$ is smooth outside the zero section $Z \subseteq \mathbb{V}(\mathcal{U})$. On the other hand $\check{\mathcal{U}}$ is globally generated, thus we have a diagram

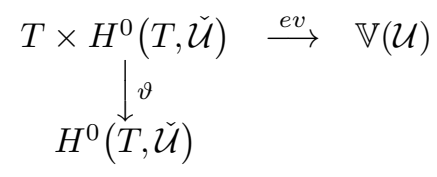

where $e v$ is smooth and it preserves codimensions. We set $\widetilde{\mathcal{D}}_{t r}:=e v^{-1}\left(C \mathcal{D}_{t r}\right)$, $\widetilde{Z}:=e v^{-1}(Z)$.

Since $\operatorname{dim}(T) \leq 7$ and $Z$ has codimension 8 , it follows that $\Gamma_{0}:=\vartheta(\widetilde{Z}) \subseteq$ $H^{0}(T, \check{\mathcal{U}})$ is a proper closed subset. The properness of $\vartheta$ and the generic smoothness theorem (see Corollary III.10.7 of [12]) applied to $\widetilde{\vartheta}:=\vartheta_{\mid \widetilde{\mathcal{D}} \backslash \widetilde{Z}}$ yields the existence of a second proper closed subset $\Gamma_{1} \subseteq H^{0}(T, \check{\mathcal{U}})$ outside of which $\widetilde{\vartheta}$ is smooth. The scheme $\vartheta^{-1}(\varphi)$ then intersects transversally $\widetilde{\mathcal{D}}$ and does not intersect $\widetilde{Z}$ for each $\varphi \in H^{0}(T, \check{\mathcal{U}}) \backslash\left(\Gamma_{0} \cup \Gamma_{1}\right)$.

Since $e v(t, \varphi)=\varphi(t)$ for each $(t, \varphi) \in T \times H^{0}(T, \check{\mathcal{U}})$, it follows that the projection $T \times H^{0}(T, \check{\mathcal{U}}) \rightarrow T$ gives $\vartheta^{-1}(\varphi) \cap \widetilde{\mathcal{D}}_{t r} \cong D_{t r}(\varphi)$, hence the last scheme is smooth for a general choice of $\varphi \in H^{0}(T, \check{\mathcal{U}})$.

Now let $Y$ be an integral scheme, choose three locally free $\mathcal{O}_{Y}$-sheaves of rank $2, \mathcal{A}_{i}, 1 \leq i \leq 3$ and let $\mathcal{A}:=\mathcal{A}_{1} \otimes \mathcal{A}_{2} \otimes \mathcal{A}_{3}$ and $\bar{\pi}: \overline{\mathbb{P}}:=\mathbb{P}(\mathcal{A}) \rightarrow Y$ be the projection. Thus we have the totally degeneracy locus $\mathcal{D}_{t r} \subseteq \overline{\mathbb{P}}$. 
Consider now a locally free $\mathcal{O}_{Y}$-sheaf $\mathcal{E}$ of rank 5: set $\mathbb{P}:=\mathbb{P}(\mathcal{E})$ and denote by $\pi: \mathbb{P} \rightarrow Y$ the canonical projection. Via the projection isomorphism $\Phi: H^{0}(Y, \check{\mathcal{A}} \otimes \mathcal{E}) \stackrel{\sim}{\longrightarrow} H^{0}\left(\mathbb{P}, \pi^{*} \check{\mathcal{A}}(1)\right)$, each $\delta \in H^{0}\left(\mathbb{P}, \pi^{*} \check{\mathcal{A}}(1)\right)$ induces $\eta:=\Phi^{-1}(\delta) \in H^{0}(Y, \check{\mathcal{A}} \otimes \mathcal{E})$ hence a map $i: \mathbb{P} \hookrightarrow \overline{\mathbb{P}}$.

Assume that $\eta$ induces a surjective map from $\mathcal{A}$ to $\mathcal{E}$ : then $i$ is an embedding and the sheaf of ideals of $i^{-1}\left(\mathcal{D}_{t r}\right) \cong i(\mathbb{P}) \cap \mathcal{D}_{t r}$ and $D_{t r}(\delta)$ have the same local generators, thus the two schemes coincide. Since $\mathcal{D}_{t r}, \mathbb{P}$ and $\overline{\mathbb{P}}$, fibrewise over $Y$, have respective dimensions 3,4 and 7 , it follows that fibrewise $D_{t r}(\delta)$ has dimension at least 0 (in particular, $D_{t r}(\delta)$ is fibrewise, hence globally, nonempty). Let $\varrho:=\pi_{\mid X}$.

Definition 2.6. With the notations above we say that $\eta$ has the right codimension at $y \in Y$ if the scheme $X_{y}:=\varrho^{-1}(y) \subseteq \pi^{-1}(y)=: \mathbb{P}_{y}$ has codimension 4 .

If $\eta$ has the right codimension at each point $y \in Y$, then $\varrho$ is finite. Moreover, Lemma 2.2 also yields that $\varrho$ is fibrewise Gorenstein of degree 6 , whence $\varrho: X \rightarrow$ $Y$ is a Gorenstein cover of degree $d=6$. Such a kind of cover will be called Anglo-American.

The reason for this terminology is that while for Scandinavian covers the minimal free resolution of $\mathcal{O}_{X_{y}}$ as sheaf of modules over $\mathcal{O}_{\mathbb{P}_{y}} \cong \mathcal{O}_{\mathbb{P}_{k}^{4}}$ is related to the Gulliksen-Negård complex, called Scandinavian following [16], for Anglo-American covers the above mentioned resolution is a generalized EagonNorthcott complex as explained in Section 2 (in [14] its description in terms of cubic arrays has been ascribed to RF́ossum):

Proposition 2.7. Assume $\eta$ has the right codimension at each point $y \in Y$ and that $\operatorname{det}(\mathcal{E}) \cong\left(\operatorname{det}\left(\mathcal{A}_{1}\right) \otimes \operatorname{det}\left(\mathcal{A}_{3}\right) \otimes \operatorname{det}\left(\mathcal{A}_{3}\right)\right)^{3}$. Then $\mathcal{E}$ is the Tschirnhausen module of $\varrho$.

Proof. $\mathcal{D}_{t r}$ is fibrewise arithmetically Gorenstein in $\overline{\mathbb{P}}$ by Lemma 2.2. Since $\eta$ has the right codimension at each point $y \in Y$ the same is true for the embedding $X:=\mathcal{D}_{t r} \cap \mathbb{P} \subseteq \mathbb{P}$. Thus Theorem 2.1 of [7] yields the existence of a unique exact sequence $\mathcal{N}_{*}$

$$
\begin{aligned}
0 \longrightarrow \pi^{*} \mathcal{F}_{3}(-6) & \longrightarrow \pi^{*} \mathcal{F}_{2}(-4) \longrightarrow \pi^{*} \mathcal{F}_{1}(-3) \longrightarrow \\
& \longrightarrow \pi^{*} \mathcal{F}_{0}(-2) \longrightarrow \mathcal{O}_{\mathbb{P}} \longrightarrow \mathcal{O}_{X} \longrightarrow 0
\end{aligned}
$$

where $\mathcal{F}_{i}, 0 \leq i \leq 3$, are locally free $\mathcal{O}_{Y}$-sheaves of respective ranks $9,16,9$, 1 , whose restriction to each fibre is isomorphic to the minimal free resolution (2.2.1). Moreover, up to a unique isomorphism, $\mathcal{N}_{*}$ is the unique exact sequence enjoying such a property and $\mathcal{E}$ is Tschirnhausen module of $\varrho$ if and only if $\operatorname{det}(\mathcal{E}) \cong \mathcal{F}_{3}$. Thus it suffices to prove that

$$
\mathcal{F}_{3} \cong \mathcal{L}:=\left(\operatorname{det}\left(\mathcal{A}_{1}\right) \otimes \operatorname{det}\left(\mathcal{A}_{3}\right) \otimes \operatorname{det}\left(\mathcal{A}_{3}\right)\right)^{3} \cong \operatorname{det}(\mathcal{E}) .
$$

Let $\bar{\pi}: \overline{\mathbb{P}}:=\mathbb{P}(\mathcal{A}) \rightarrow Y$ be the projection. We have

$\omega_{\mathcal{D}_{t r} \mid \overline{\mathbb{P}}} \cong \omega_{\mathcal{D}_{t r} \mid Y} \otimes \omega_{\overline{\mathbb{P}} \mid Y}^{-1} \cong \mathcal{O}_{\overline{\mathbb{P}}}(6) \otimes \bar{\pi}^{*}\left(\operatorname{det}\left(\mathcal{A}_{1}\right) \otimes \operatorname{det}\left(\mathcal{A}_{3}\right) \otimes \operatorname{det}\left(\mathcal{A}_{3}\right)\right)^{-3} \otimes \mathcal{O}_{\mathcal{D}_{t r}}$. 
Since $X:=\mathcal{D}_{t r} \cap i(\mathbb{P}) \cong \mathcal{D}_{t r} \times_{\mathbb{P}} \mathbb{P}$, it follows that $\omega_{X \mid \mathbb{P}} \cong \omega_{\mathcal{D}_{t r} \mid \overline{\mathbb{P}}} \otimes \mathcal{O}_{X} \cong \mathcal{O}_{\overline{\mathbb{P}}}(6) \otimes \bar{\pi}^{*}\left(\operatorname{det}\left(\mathcal{A}_{1}\right) \otimes \operatorname{det}\left(\mathcal{A}_{3}\right) \otimes \operatorname{det}\left(\mathcal{A}_{3}\right)\right)^{-3} \otimes \mathcal{O}_{X}$.

Thus we also have the exact complex

$$
\begin{aligned}
0 \longrightarrow \pi^{*} \mathcal{L}(-6) & \longrightarrow \pi^{*}\left(\check{\mathcal{F}}_{0} \otimes \mathcal{L}\right)(-4) \longrightarrow \pi^{*}\left(\check{\mathcal{F}}_{1} \otimes \mathcal{L}\right)(-3) \longrightarrow \\
& \longrightarrow \pi^{*}\left(\check{\mathcal{F}}_{2} \otimes \mathcal{L}\right)(-2) \longrightarrow\left(\check{\mathcal{F}}_{3} \otimes \mathcal{L}\right) \longrightarrow \mathcal{O}_{X} \longrightarrow 0
\end{aligned}
$$

which coincides fibrewise with the sequence (2.2.1) too.

It follows that sequences (2.7.1) and (2.7.2) are isomorphic thus $\pi^{*} \mathcal{F}_{3} \cong \pi^{*} \mathcal{L}$. By applying $\pi_{*}$ it then follows that $\mathcal{F}_{3} \cong \mathcal{L}$.

Remark 2.8. It follows from Proposition 2.7 that, if $\mathcal{E}$ is the Tschirhausen module of an Anglo-American cover $\varrho: X \rightarrow Y$, then $\operatorname{det}(\mathcal{E}) \in \operatorname{Pic}(Y)$ is divisible by three.

\section{A Bertini theorem}

In this section we will prove a Bertini theorem for Anglo-American covers $\varrho: X \rightarrow Y$ of degree $d=6$, when $Y$ is a smooth and irreducible curve or surface.

Theorem 3.1. Let $k:=\mathbb{C}, Y$ be an integral, smooth projective scheme over $k$ with $\operatorname{dim}(Y) \leq 2$ and $\mathcal{E}, \mathcal{A}_{i}, 1 \leq i \leq 3$, locally free $\mathcal{O}_{Y}$-sheaves of ranks 5,2 , 2,2 respectively such that $\left(\operatorname{det}\left(\mathcal{A}_{1}\right) \otimes \operatorname{det}\left(\mathcal{A}_{3}\right) \otimes \operatorname{det}\left(\mathcal{A}_{3}\right)\right)^{3}=\operatorname{det}(\mathcal{E})$.

If $\mathcal{A}:=\mathcal{A}_{1} \otimes \mathcal{A}_{3} \otimes \mathcal{A}_{3}$ is globally generated the sets,

$H_{r c}:=\left\{\eta \in H^{0}(Y, \check{\mathcal{A}} \otimes \mathcal{E}) \mid \eta\right.$ has the right codimension at each $\left.y \in Y\right\}$,

$H_{s}:=\left\{\eta \in H_{r c} \mid D_{t r}(\Phi(\eta)) \subseteq \mathbb{P}\right.$ is smooth $\}$

are open and non-empty. $D_{t r}(\Phi(\eta))$ is connected if and only if $h^{0}(Y, \check{\mathcal{E}})=0$.

Proof. If $\operatorname{dim}(Y) \leq 4$, each general section $\eta \in H^{0}(Y, \check{\mathcal{A}} \otimes \mathcal{E})$ corresponds to an epimorphism $\eta: \mathcal{A} \rightarrow \mathcal{E}$, hence to a linear embedding $j: \mathbb{P} \hookrightarrow \overline{\mathbb{P}}:=\mathbb{P}(\mathcal{A})$ such that $X:=D_{t r}(\Phi(\eta)) \cong \mathcal{D}_{t r} \cap \mathbb{P}$. The map $\varrho:=\pi_{\mid X}$ is a cover if and only if $\operatorname{dim}\left(\mathcal{D}_{t r} \cap \mathbb{P}_{y}\right)=0$ for each $y \in Y$.

Since $\breve{\mathcal{A}} \otimes \mathcal{E}$ is globally generated the evaluation map induces an epimorphism $H^{0}(Y, \check{\mathcal{A}} \otimes \mathcal{E}) \otimes k(y) \rightarrow \check{\mathcal{A}} \otimes \mathcal{E} \otimes k(y)$. Since $\check{\mathcal{A}} \otimes \mathcal{E} \cong \pi_{*}\left(\pi^{*} \check{\mathcal{A}}(1)\right)$, then Grauert's theorem (see [12], Corollary III.12.9) yields the surjectivity of

$$
\alpha_{y}: H^{0}\left(\mathbb{P}, \pi^{*} \check{\mathcal{A}}(1)\right) \rightarrow H^{0}\left(\mathbb{P}_{y}, j_{y}^{*} \pi^{*} \check{\mathcal{A}}(1)\right) \cong H^{0}\left(\mathbb{P}_{k}^{4}, \mathcal{O}_{\mathbb{P}_{k}^{4}}(1)\right)^{\oplus 8} .
$$

On the other hand we have a surjective rational map $\beta: H^{0}\left(\mathbb{P}_{k}^{4}, \mathcal{O}_{\mathbb{P}_{k}^{4}}(1)\right)^{\oplus 8} \rightarrow$ $G(5,8)$ (in the paper $G(m, n)$ denotes the Grassmann variety of $m$-subspaces of $k^{n}$ ), hence a surjective rational map

$$
\gamma_{y}: H^{0}(Y, \check{\mathcal{A}} \otimes \mathcal{E}) \cong H^{0}\left(\mathbb{P}, \pi^{*} \check{\mathcal{A}}(1)\right) \rightarrow G(5,8) .
$$


From now on $\mathcal{D}_{t r}$ will denote the image of the Segre embedding $\mathbb{P}_{k}^{1} \times \mathbb{P}_{k}^{1} \times \mathbb{P}_{k}^{1} \hookrightarrow$ $\mathbb{P}_{k}^{7}$. Let

$$
F:=\left\{h \in G(5,8) \mid \operatorname{dim}\left(h \cap \mathcal{D}_{t r}\right) \geq 1\right\}
$$

(For a closed scheme $T$ we denote by $\operatorname{dim}(T)$ the maximum of the dimensions of its irreducible components).

Consider the correspondence

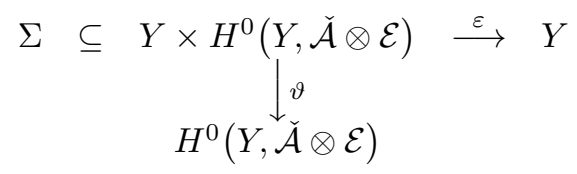

where $\Sigma:=\{(y, \eta) \mid \eta$ has not the right codimension at $y\} . \quad \Sigma$ is closed (see the analogous step in the proof of Theorem 3.6 of [7]) and the elements of $\Sigma_{y}:=\Sigma \cap \varepsilon^{-1}(y)$ are the pairs $(y, \eta)$ such that $\delta:=\Phi(\eta)$ specializes at $y$ via $\alpha_{y}$ to a 4 -space $h \subseteq \mathbb{P}(H) \cong \mathbb{P}_{k}^{7}$ such that $\operatorname{dim}\left(h \cap \mathcal{D}_{t r}\right) \geq 1$. It follows that $\Sigma_{y}=y \times \gamma_{y}^{-1}(F)$.

Claim 3.2. $\operatorname{dim}(F) \leq 12$, hence the codimension of $F$ inside $G(5,8)$ is at least 3 .

Assume the claim. Then the codimension of $\Sigma$ is at least 3 thus the codimension of $\vartheta(\Sigma)$ is at least 1 since $\operatorname{dim}(Y) \leq 2$.

Since $\operatorname{dim}(Y) \leq 2$, it follows that $\operatorname{dim}(\mathbb{P}) \leq 6$. Since $\check{\mathcal{A}} \otimes \mathcal{E}$ is globally generated, the same is true for $\pi^{*} \check{\mathcal{A}}(1)$. It follows that Proposition 2.5 with $T=\mathbb{P}$ and $\mathcal{U}_{1}:=\pi^{*} \mathcal{A}_{1}(-1), \mathcal{U}_{2}:=\pi^{*} \mathcal{A}_{2}, \mathcal{U}_{3}:=\pi^{*} \mathcal{A}_{3}$ yields that $D_{t r}(\Phi(\eta))$ is smooth for a general $\eta \in H^{0}(Y, \check{\mathcal{A}} \otimes \mathcal{E})$.

Formula (1.1) implies that $h^{0}\left(X, \mathcal{O}_{X}\right)=h^{0}\left(Y, \mathcal{O}_{Y}\right)+h^{0}(Y, \check{\mathcal{E}})$ hence for each section $\eta \in H^{0}(Y, \check{\mathcal{A}} \otimes \mathcal{E}), X:=D_{t r}(\Phi(\eta))$ is connected if and only if $h^{0}(Y, \check{\mathcal{E}})=0$.

In order to complete the proof of Theorem 3.1 we have to prove Claim 3.2. To this purpose we will denote by $\mathrm{Hilb}_{n, p}$ the closure in the corresponding Hilbert scheme of the locus of integral subschemes $C \subseteq \mathcal{D}_{t r}$ having Hilbert polynomial $P_{C}(t)=n t+1-p$. For generalities about Hilbert schemes see [11] or [19], Lecture 15. Consider the incidence correspondence

$$
\mathcal{I}_{n}^{p}:=\left\{(h, C) \in G(5,9) \times \operatorname{Hilb}_{n, p} \mid C \subseteq h\right\}
$$

and let $F_{n, p}$ be the closure of its projection onto $G(5,9)$. By semicontinuity $\operatorname{dim}\left(h \cap \mathcal{D}_{t r}\right) \geq 1$ for $h \in F_{n, p}$.

Let $h=: h_{3} \subset h_{2} \subset h_{1} \subset h_{0}:=\mathbb{P}(H) \cong \mathbb{P}_{k}^{7}$ be a flag of subspaces containing $h \in F$. Then $\mathcal{D}_{t r} \cap h \subset \mathcal{D}_{t r} \cap h_{2} \subset \mathcal{D}_{t r} \cap h_{1} \subset \mathcal{D}_{t r}$. In order to have $\operatorname{dim}\left(\mathcal{D}_{t r} \cap h\right) \geq 1$ there must exists $i$ such that $\operatorname{dim}\left(\mathcal{D}_{t r} \cap h_{i}\right)=\operatorname{dim}\left(\mathcal{D}_{t r} \cap h_{i+1}\right)$, and we take the first of such indices. 
The minimal free resolution of the ideal sheaf $\Im_{\mathcal{D}_{t r}}$ of $\mathcal{D}_{t r}$ in $\mathcal{O}_{\mathbb{P}_{k}^{7}}$ is (see Lemma 2.2)

$$
0 \rightarrow \mathcal{O}_{\mathbb{P}_{k}^{7}}(-6) \rightarrow \mathcal{O}_{\mathbb{P}_{k}^{7}}(-4)^{\oplus 9} \rightarrow \mathcal{O}_{\mathbb{P}_{k}^{7}}(-3)^{\oplus 16} \rightarrow \mathcal{O}_{\mathbb{P}_{k}^{7}}(-2)^{\oplus 9} \rightarrow \Im_{\mathcal{D}_{t r}} \rightarrow 0 .
$$

Since $\mathcal{D}_{t r} \cap h_{i}$ is complete intersection inside $\mathcal{D}_{t r}$, tensoring the above sequence by $\mathcal{O}_{h_{i}}$ we obtain the minimal free resolution of $\Im_{\mathcal{D}_{t r} \cap h_{i}}$ in $\mathcal{O}_{h_{i}}$.

It follows $h^{0}\left(h_{i}, \Im_{\mathcal{D}_{t r} \cap h_{i}}(1)\right)=0$ whence $\mathcal{D}_{t r} \cap h_{i} \nsubseteq h_{i+1}$, and there is a proper component of $\mathcal{D}_{t r} \cap h_{i}$, say $\widehat{T}$, contained in $h_{i+1}$. Since $\operatorname{deg}\left(\mathcal{D}_{t r}\right)=6$, it follows that $\operatorname{deg}(\widehat{T}) \leq 5$. Thus $h \in F$ if and only if $h \cap \mathcal{D}_{t r}$ contains an integral, closed subscheme $T$ (namely any component of $h \cap \widehat{T}$ ), such that $\operatorname{deg}(T) \leq 5$ contained in $h \cong \mathbb{P}_{k}^{4}$ in an intersection of hyperplanes and quadrics. Thus, if $\operatorname{dim}(T)=1$, then $h \in F_{n, p}$ with $n \leq 5$. The above description and Castelnuovo's bound (see page 116 of [2]) yield $p \leq 1$.

If $\operatorname{dim}(T) \geq 2$, cutting with hyperplanes in $h$ we can always reduce to the case $\operatorname{dim}(T)=2$. If this is the case, each irreducible component of $T$ must be either a quadric in a hyperplane of $h$, or a non-degenerate cubic (hence a scroll), or a complete intersection of two hyperquadrics (hence a generalized del Pezzo surface). In any case $T$ contains a line, thus $h \in 1,0$.

Hence

$$
F=F_{1,0} \cup F_{2,0} \cup F_{3,0} \cup F_{4,0} \cup F_{4,1} \cup F_{5,0} \cup F_{5,1} \subseteq G(5,8) .
$$

We now prove Claim 3.2 checking that $\operatorname{dim}\left(F_{n, p}\right) \leq 12$ for each $n$ and $p$ considered in the list above. We begin by dealing with $F_{n, p}$ and bounding its dimension. Let $G_{n, p}:=\{(A, h) \mid A \in h\} \subseteq \mathcal{D}_{t r} \times F_{n, p}$ and consider the map $g_{n, p}: G_{n, p} \rightarrow F_{n, p}$ induced by the projection on the second factor. $g_{n, p}$ is surjective and its fibres have dimension at least 1 , hence $\operatorname{dim}\left(F_{n, p}\right) \leq \operatorname{dim}\left(G_{n, p}\right)-1$. On the other hand we have also the map $f_{n, p}: G_{n, p} \rightarrow \mathcal{D}_{t r}$ induced by the projection on the first factor.

Since $\mathcal{D}_{t r}$ is homogeneous with respect to the action of $G=S L_{2} \times S L_{2} \times S L_{2}$, $f_{n, p}$ is surjective too, so $\operatorname{dim}\left(G_{n, p}\right)=3+\operatorname{dim}\left(f_{n, p}^{-1}(E)\right)$, whence $\operatorname{dim}\left(F_{n, p}\right) \leq$ $2+\operatorname{dim}\left(f_{n, p}^{-1}(E)\right), E:=(1,0, \ldots, 0)$.

3.3. $\boldsymbol{F}_{\mathbf{1}, \mathbf{0}}$. The scheme Hilb $\mathrm{b}_{1,0}$ is well-known (see Proposition 3.5.6 of [13] and the reference therein): we give here its description for sake of completeness. Consider a line $r \subseteq \mathbb{P}(H) \cong \mathbb{P}_{k}^{7}$ through $E:=(1,0, \ldots, 0)$. Its parametric equations are of the form

$$
U=\lambda E+\mu A,
$$

where $A:=\left(a_{i, j, h}\right)$. By a linear change of the parameters $\lambda, \mu$ we can always assume that $a_{0,0,0}=0$. Since $r \subseteq \mathcal{D}_{t r}$ we must have by equations (2.2.2)

$$
0=u_{0,0,0} u_{0,1,1}-u_{0,1,0} u_{0,0,1}=a_{0,1,1} \lambda \mu-a_{0,1,0} a_{0,0,1} \mu^{2}
$$

for each $\lambda, \mu \in k$, whence $a_{0,1,1}=0$. Analogously $a_{1,0,1}=a_{1,1,0}=a_{1,1,1}=0$. In particular, we must also have

$$
a_{0,1,0} a_{0,0,1}=a_{1,0,0} a_{0,0,1}=a_{1,0,0} a_{0,1,0}=0,
$$


which are equivalent to the three conditions $a_{0,1,0}=a_{0,0,1}=0, a_{1,0,0}=a_{0,0,1}=$ 0 and $a_{1,0,0}=a_{0,1,0}=0$.

This means that through $E$ there are only three lines. Since the set of $4-$ spaces $h \subseteq \mathbb{P}_{k}^{7}$ containing a fixed line is isomorphic to $G(3,6)$. It follows that $\operatorname{dim}\left(f_{1,0}^{-1}(E)\right)=\operatorname{dim}(G(3,6))=9$, whence $\operatorname{dim}\left(F_{1,0}\right) \leq 11$.

3.4. $\boldsymbol{F}_{\mathbf{2}, \mathbf{0}}$. Each point of $\mathrm{Hilb}_{2,0}$ is either an integral conic or decomposes in lines: this second case has been already examined in 3.3, so we can restrict ourselves to consider an integral conic $C \subseteq \mathbb{P}(H) \cong \mathbb{P}_{k}^{7}$ through $E$. Its parametric equations are of the form

$$
U=\lambda^{2} E+\lambda \mu A+\mu^{2} B
$$

where $U:=\left(u_{i, j, h}\right), A:=\left(a_{i, j, h}\right), B:=\left(b_{i, j, h}\right)$. By a linear change of the parameters $\lambda, \mu$ we can always assume that $a_{0,0,0}=0$.

If $C \subseteq \mathcal{D}_{t r}$, then we must have by equations $(2.2 .2)$

$$
\begin{aligned}
0 & =u_{0,0,0} u_{0,1,1}-u_{0,1,0} u_{0,0,1} \\
& =a_{0,1,1} \lambda^{3} \mu+\left(b_{0,1,1}-a_{0,1,0} a_{0,0,1}\right) \lambda^{2} \mu^{2}+\text { terms in } \lambda \mu^{3}, \mu^{4}
\end{aligned}
$$

for each $\lambda, \mu \in k$. Analogous identities hold for the other equations of $\mathcal{D}_{t r}$ containing $u_{0,0,0}$. Hence $a_{0,1,1}=a_{1,0,1}=a_{1,1,0}=a_{1,1,1}=0$ : let us denote by $H_{a}:=\left\{a_{0,0,0}=a_{0,1,1}=a_{1,0,1}=a_{1,1,0}=a_{1,1,1}=0\right\} \subseteq H$. Moreover, we also have

$$
b_{0,1,1}-a_{0,1,0} a_{0,0,1}=b_{1,0,1}-a_{1,0,0} a_{0,0,1}=b_{1,1,0}-a_{1,0,0} a_{0,1,0}=0 .
$$

Analogously looking at the coefficient of $\lambda^{2} \mu^{2}$ in $u_{0,0,0} u_{1,1,1}-u_{1,0,0} u_{0,1,1}$ we obtain $b_{1,1,1}=0$.

Consider

$$
Z:=\left\{(A, B) \in H_{a} \times H \mid T=\lambda^{2} E+\lambda \mu A+\mu^{2} B \text { satisfies (3.4.2) }\right\},
$$

and its natural projection $p: Z \rightarrow$ Hilb $_{2,0}$ onto the locus of conics through $E$.

Since $B$ satisfies equations (2.2.2) (look at the coefficients of $\mu^{4}$ ), it follows that the image of the projection $q: Z \rightarrow H$ on the second factor is contained in the intersection of the cone $C \mathcal{D}_{t r} \subseteq H$ over $\mathcal{D}_{t r} \subseteq \mathbb{P}(H) \cong \mathbb{P}_{k}^{7}$ with the hyperplane $\left\{b_{1,1,1}=0\right\} \subseteq H$, which has dimension 3 .

Equations (3.4.2) easily yield $\operatorname{dim}\left(q^{-1}(B)\right) \leq 1$, whence $\operatorname{dim}(Z) \leq 4$. Since $p$ is equivariant with respect to the action $(A, B) \rightarrow\left(\mu A, \mu^{2} B\right)$ of $k^{*}$ onto $Z$, it follows that the locus of conics through $E$ has dimension at most 3 . Since the set of 4 -spaces $h \subseteq \mathbb{P}_{k}^{7}$ containing a fixed integral conic is isomorphic to $G(2,5)$ we conclude that $\operatorname{dim}\left(f_{1,0}^{-1}(E)\right) \leq 9$, whence $\operatorname{dim}\left(F_{2,0}\right) \leq 11$.

3.5. $\boldsymbol{F}_{\mathbf{3}, \mathbf{0}}$. Each point of Hilb $\mathrm{b}_{3,0}$ is either a smooth, integral and rational cubic or it contains a line or a conic. Again we can restrict to the first case. Consider such a cubic $D \subseteq \mathbb{P}_{k}^{7}$ through $E$. Its parametric equations are

$$
U=\lambda^{3} E+\lambda^{2} \mu A+\lambda \mu^{2} B+\mu^{3} C,
$$


where $U:=\left(u_{i, j, h}\right), A:=\left(a_{i, j, h}\right), B:=\left(b_{i, j, h}\right), C:=\left(c_{i, j, h}\right)$. Again we assume that $a_{0,0,0}=0$.

If $D \subseteq \mathcal{D}_{t r}$, looking at the equations (2.2.2) involving $u_{0,0,0}$, we obtain $a_{0,1,1}=a_{1,0,1}=a_{1,1,0}=a_{1,1,1}=0$ (coefficients of $\left.\lambda^{5} \mu\right), b_{1,1,1}=0$ and

$$
b_{0,1,1}-a_{0,1,0} a_{0,0,1}=b_{1,0,1}-a_{1,0,0} a_{0,0,1}=b_{1,1,0}-a_{1,0,0} a_{0,1,0}=0
$$

(coefficients of $\lambda^{4} \mu^{2}$ ),

$$
\begin{gathered}
c_{0,1,1}-a_{0,0,1} b_{0,1,0}-a_{0,1,0} b_{0,0,1}=0 \\
c_{1,0,1}-a_{0,0,1} b_{1,0,0}-a_{1,0,0} b_{0,0,1}=0 \\
c_{1,1,0}-a_{0,1,0} b_{1,0,0}-a_{1,0,0} b_{0,1,0}=0, \\
c_{1,1,1}-a_{1,0,0} b_{0,1,1}=0
\end{gathered}
$$

(coefficients of $\lambda^{3} \mu^{3}$ ). Finally $D$ satisfies the equations (2.2.2) (coefficients of $\left.\mu^{4}\right)$.

Let $H_{a}$ be as in the previous section and $H_{b}:=\left\{b_{1,1,1}=0\right\}$. Consider

$$
\begin{aligned}
Z:=\{ & (A, B, C) \in H_{a} \times H_{b} \times H \mid T=\lambda^{3} E+\lambda^{2} \mu A+\lambda \mu^{2} B+\mu^{3} C \\
& \text { satisfies }(3.5 .1),(3.5 .2),(3.5 .3)\},
\end{aligned}
$$

and $p: Z \rightarrow$ Hilb $_{3,0}$ the projection on the locus of cubics through $E$. The image of the projection $q: Z \rightarrow H$ on the third factor is the cone $C \mathcal{D}_{t r} \subseteq H$ over $\mathcal{D}_{t r} \subseteq \mathbb{P}(H) \cong \mathbb{P}_{k}^{7}$, which has dimension 4. Thus $R:=\left\{C \in \operatorname{im}(q) \mid c_{1,1,1}=0\right\}$ satisfies $\operatorname{dim}(R) \leq 3$.

Equations (3.5.1) and (3.5.3) yield

$$
c_{1,1,1}-a_{1,0,0} a_{0,1,0} a_{0,0,1}=0 .
$$

Take $C \notin R$. It follows that $a_{1,0,0} a_{0,1,0} a_{0,0,1} \neq 0$, thus the matrix of the linear system (3.5.2) in the variables $b_{0,1,0}, b_{0,0,1}, b_{1,0,0}$ is non-singular, hence any choice of $A$ satisfying (3.5.4) gives all the $b_{i, j, h}$ when $(i, j, h) \neq(0,0,0)$. It follows that $\operatorname{dim}\left(q^{-1}(C)\right) \leq 2$ in this case.

Now let $C \in R$. Consider the map $\widehat{q}: q^{-1}(C) \rightarrow H_{a}$ induced by the projection on the first factor: its image is the union of the coordinates planes. If $a_{1,0,0}=0$ but $a_{0,1,0} a_{0,0,1} \neq 0$, then the matrix of (3.5.2) has rank 2, thus $\operatorname{dim}\left(\widehat{q}^{-1}(A)\right) \leq 1$. If $a_{1,0,0}=a_{0,1,0}=0$ but $a_{0,0,1} \neq 0$, then the matrix of (3.5.2) has rank 1 , thus $\operatorname{dim}\left(\widehat{q}^{-1}(A)\right) \leq 2$. Finally, if $A=0$, then $\operatorname{dim}\left(\widehat{q}^{-1}(A)\right) \leq 3$. We conclude that $\operatorname{dim}\left(q^{-1}(C)\right) \leq 3$.

From the above analysis it follows that $\operatorname{dim}(Z) \leq 7$. Again $p$ is equivariant with respect to the action $(A, B, C) \rightarrow\left(\mu A, \mu^{2} B, \mu^{3} C\right)$ of $k^{*}$ onto $Z$, then the locus of cubics through $E$ has dimension at most 6 . Since the set of 4 -spaces $h \subseteq \mathbb{P}_{k}^{7}$ containing a fixed smooth, integral, rational cubic is isomorphic to $G(1,4)$ we conclude that $\operatorname{dim}\left(f_{1,0}^{-1}(E)\right) \leq 6$, whence $\operatorname{dim}\left(F_{3,0}\right) \leq 11$.

3.6. $\boldsymbol{F}_{\mathbf{4}}:=\boldsymbol{F}_{\mathbf{4}, \mathbf{0}} \cup \boldsymbol{F}_{\mathbf{4}, \mathbf{1}}$. Recall that if, for each $h \in F_{4}$, we have $\operatorname{dim}\left(h \cap \mathcal{D}_{t r}\right) \geq$ 2 , then $F_{4} \subseteq F_{1,0}$. Thus we assume that $\operatorname{dim}\left(h \cap \mathcal{D}_{t r}\right)=1$ for $h$ in a suitable open subset of $F_{4}$. Let $\widehat{F}_{4}$ be its closure inside $F_{4}$. 
It follows that each general 5-space $h_{5} \subseteq \mathbb{P}(H) \cong \mathbb{P}_{k}^{7}$ containing a general $h \in \widehat{F}_{4}$ is such that $h_{5} \cap \mathcal{D}_{t r}=C \cup C^{\prime}$ where $C$ is an integral quartic and $\operatorname{deg}\left(C^{\prime}\right)=2$. We can distinguish two cases. Either for each integral quartic $C$ and 5 -space $h_{5}$ the residue curve $C^{\prime}$ is a union of lines or there is one integral quartic $C$ and a 5 -space $h_{5}$ such that $C^{\prime}$ is an integral conic.

In the first case consider in Hilb H, $_{1,0} \times$ Hilb $_{1,0} \times \widehat{F}_{4} \times G(6,8)$ the subset

$$
\mathcal{I}:=\left\{\left(r_{1}, r_{2}, h, h_{5}\right) \mid h_{5} \cap \mathcal{D}_{t r}=C \cup r_{1} \cup r_{2} \text { and } C \subseteq h \subseteq h_{5}\right\} .
$$

We have the projection onto $F_{4}$, thus $\operatorname{dim}\left(\widehat{F}_{4}\right) \leq \operatorname{dim}(\mathcal{I})$. On the other hand we have also the projection $\alpha: \mathcal{I} \rightarrow \operatorname{Hilb}_{1,0} \times \operatorname{Hilb}_{1,0}$ and we bound $\operatorname{dim}(\mathcal{I})$ by computing the dimension of the fibres of $\alpha$. Take $\left(r_{1}, r_{2}\right) \in$ Hilb $_{1,0} \times$ Hilb $_{1,0}$.

If the two lines are skew, then the set of 5-spaces containing $r_{1} \cup r_{2}$ is isomorphic to $G(2,4)$. Fix such an $h_{5}$ and assume that $h_{5} \cap \mathcal{D}_{t r}=C \cup r_{1} \cup$ $r_{2}$. Since $C$ is intersection of quadrics, it follows that it generates a space of dimension at least 3 . Thus the set of $h \in \widehat{F}_{4}$ containing such a $C$ and contained in $h_{5}$ has dimension at most 1 . It follows that $\operatorname{dim}\left(\alpha^{-1}\left(r_{1} \cup r_{2}\right)\right) \leq 5$. On the other hand $\operatorname{dim}\left(\mathrm{Hilb}_{1,0} \times \mathrm{Hilb}_{1,0}\right)=4$.

If the two lines are incident, then the set of 5 -spaces containing $r_{1} \cup r_{2}$ is isomorphic to $G(3,5)$. As in the previous case one checks that $\operatorname{dim}\left(\alpha^{-1}\left(r_{1} \cup\right.\right.$ $\left.\left.r_{2}\right)\right) \leq 7$. On the other hand $\operatorname{dim}\left(\left\{\left(r_{1}, r_{2}\right) \in \mathrm{Hilb}_{1,0} \times \mathrm{Hilb}_{1,0} \mid r_{1} \cap r_{2} \neq \emptyset\right\}\right)=3$. We conclude that in any case $\operatorname{dim}(\mathcal{I}) \leq 10$.

Consider the second case. In the Chow ring $A(G(5,8))$ the scheme $\widehat{F}_{4}$ is equivalent to

$$
\begin{aligned}
a \sigma_{(0,0,0,0,0)} & +b \sigma_{(1,0,0,0,0)}+c \sigma_{(2,0,0,0,0)}+d \sigma_{(1,1,0,0,0)} \\
& + \text { cycles of codimension at least } 3
\end{aligned}
$$

(for the notations and the intersection theory on $G(5,9)$ we refer to Chapter 1 , Section 5 of $[10])$.

Let $h_{6} \subseteq \mathbb{P}_{k}^{7}$ be a general 6-space. Then $\operatorname{dim}\left(\mathcal{D}_{t r} \cap h_{6}\right)=2$ and we can find a 3-space $h_{3} \subseteq h_{6}$ such that $h_{3} \cap \mathcal{D}_{t r}=\emptyset$. On one hand each $h_{4} \in G(5,8)$ with $h_{3} \subseteq h_{4} \subseteq h_{6}$ intersect $\mathcal{D}_{t r}$ in a finite number of points, then the cycle

$$
W^{\prime}:=\left\{h_{4} \in G(5,8) \mid h_{3} \subseteq h_{4} \subseteq h_{6}\right\}
$$

does not intersect $\widehat{F}_{4}$. On the other hand $W^{\prime}$ is equivalent in the Chow ring $A(G(5,8))$ to $\sigma_{(4,4,4,4,2)}$. Taking into account that $0=W^{\prime} \cdot F_{4,1}=a \sigma_{(4,4,4,4,2)}+$ $b \sigma_{(4,4,4,4,3)}+c \sigma_{(4,4,4,4,4)}$, it follows that $a=b=c=0$.

Let $h_{5}$ be a 5 -space such that $h_{5} \cap \mathcal{D}_{t r}=C \cup C^{\prime}$ where $C$ is an integral quartic and $C^{\prime}$ an integral conic. Choose a general 2-space $h_{2} \subseteq h_{5}$. Then $h_{2}$ does not intersect the 2-space generated by $C^{\prime}$ and it is not contained in any 4-space containing $C$. Consider

$$
W^{\prime \prime}:=\left\{h_{4} \in G(5,8) \mid h_{2} \subseteq h_{4} \subseteq h_{5}\right\} .
$$


Since $h_{5} \cap \mathcal{D}_{t r}=C \cup C^{\prime}$, the generality of $h_{2}$ implies that $h_{4} \cap \mathcal{D}_{t r}$ is a finite number of points for each $h_{4} \in W^{\prime \prime}$. Moreover, $W^{\prime \prime}$ is equivalent in the Chow ring $A(G(5,8))$ to $\sigma_{(4,4,4,3,3)}$, hence $0=W^{\prime \prime} \cdot \widehat{F}_{4}=d \sigma_{(4,4,4,4,4)}$ yields $d=0$.

We conclude that in any case $\operatorname{dim}\left(F_{4}\right) \leq 12$.

3.7. $\boldsymbol{F}_{\mathbf{5}}:=\boldsymbol{F}_{\mathbf{5}, \mathbf{0}} \cup \boldsymbol{F}_{\mathbf{5}, \mathbf{1}}$. As above we denote by $\widehat{F}_{5}$ the closure inside $F_{5}$ of the set of $h \in F_{5}$ such that $\operatorname{dim}\left(h \cap \mathcal{D}_{t r}\right)=1$.

Let

$$
\mathcal{I}:=\left\{\left(h, h_{5}\right) \in G(5,8) \times G(6,8) \mid h \subseteq h_{5}\right\}
$$

and denote by $\alpha: \mathcal{I} \rightarrow G(5,8)$ and by $\beta: \mathcal{I} \rightarrow G(6,8)$ the two projections. One has $\alpha^{-1}(h) \cong \mathbb{P}_{k}^{2}$ and $\beta^{-1}\left(h_{5}\right) \cong \mathbb{P}_{k}^{5}$.

If $h \in \widehat{F}_{5}$ is general, then there is a line $r \in \mathrm{Hilb}_{1,0}$ and a 5 -space $h_{5}$ containing $h$ such that $h_{5} \cap \mathcal{D}_{t r}=C \cup r$ where $C$ is an integral quintic.

Define $G:=\left\{h_{5} \in G(6,8) \mid \exists r \in\right.$ Hilb $\left._{1,0}: r \subseteq h_{5}\right\}$. For each general $h_{5} \in G$ there exists exactly one line $r \subseteq h_{5}$, hence we have a rational dominant map $\gamma: G \rightarrow$ Hilb $_{1,0}$, whose fibre is $\gamma^{-1}(r) \cong G(4,6)$. It follows that $G$ has three components, $G^{\prime}$ dominating Hilb ${ }_{1,0}^{\prime}, G^{\prime \prime}$ dominating Hilb ${ }_{1,0}^{\prime \prime}$ and $G^{\prime \prime \prime}$ dominating Hilb ${ }_{1,0}^{\prime \prime \prime}$ all of them of dimension 10 .

Let $\widehat{G}^{\prime}:=\alpha \beta^{-1}\left(G^{\prime}\right), \widehat{G}^{\prime \prime}:=\alpha \beta^{-1}\left(G^{\prime \prime}\right), \widehat{G}^{\prime \prime \prime}:=\alpha \beta^{-1}\left(G^{\prime \prime \prime}\right)$. Notice that $\alpha^{-1}(h) \subseteq \beta^{-1}\left(G^{\prime} \cup G^{\prime \prime} \cup G^{\prime \prime \prime}\right)$ for each $h \in \widehat{F}_{5}$, hence $\widehat{F}_{5} \subseteq \widehat{G}^{\prime} \cup \widehat{G}^{\prime \prime} \cup \widehat{G}^{\prime \prime \prime}$ and $\operatorname{dim}\left(\widehat{G}^{\prime}\right)=\operatorname{dim}\left(\widehat{G}^{\prime \prime}\right)=\operatorname{dim}\left(\widehat{G}^{\prime \prime \prime}\right)=13$.

Take $h_{5} \cap \mathcal{D}_{t r}=C \cup r$, where $C$ is an integral quintic on $\mathcal{D}_{t r}$ and $h^{\prime} \subseteq h_{5}$, $C \nsubseteq h^{\prime}$. Assume that $r \in$ Hilb $_{1,0}^{\prime}$, hence $h^{\prime} \in \widehat{G}^{\prime} \backslash \widehat{F}_{5}$. Since the automorphisms $\mathcal{D}_{t r} \cong \mathbb{P}_{k}^{1} \times \mathbb{P}_{k}^{1} \times \mathbb{P}_{k}^{1}$ exchanging the factors extend to the ambient space $\mathbb{P}_{k}^{7}$, we get the existence of $h^{\prime \prime} \in \widehat{G}^{\prime \prime} \backslash \widehat{F}_{5}$ and $h^{\prime \prime \prime} \in \widehat{G}^{\prime \prime \prime} \backslash \widehat{F}_{5}$.

It follows that $\operatorname{dim}\left(F_{5}\right) \leq 12$.

Claim 3.2 is now completely proved.

\section{Examples and counterexamples}

In the following section we give some easy examples of applications of Theorem 3.1 above producing surfaces covering $\mathbb{P}_{k}^{2}$.

Let $\varrho: X \rightarrow Y$ be an Anglo-American cover with invariant sheaves $\mathcal{A}_{i}$, $1 \leq i \leq 3$ and $\mathcal{E}$. Then $X=\mathcal{D}_{t r} \cap \mathbb{P}(\mathcal{E}) \subseteq \overline{\mathbb{P}}$, thus the commutative diagram

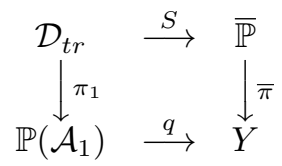

yields a factorization $\varrho=\sigma \circ \tau$ where $\tau:=\left(\pi_{1}\right)_{\mid X}: X \rightarrow S:=\pi_{\mathcal{A}_{1}}(X) \subseteq \mathbb{P}\left(\mathcal{A}_{1}\right)$ and $\sigma:=q_{\mid S}: S \rightarrow Y$.

In Section 4 of [6] we already introduced Anglo-American covers and we described such a factorization, proving in Proposition 4.5 therein that $\tau$ is always finite of degree 2. Unfortunately such a result is wrong as the following 
example shows: the general situation is that $\tau$ is birational on $S$ and $\sigma$ has degree 6 .

Example 4.1. Let $x_{0}, x_{1}, x_{2}$ be coordinates on $\mathbb{P}_{k}^{2}$ and let $A$ be the $6 \times 1$ matrix whose entries are all the monomials of degree 2 in the $x_{i}$ 's in some fixed order. Thus we have an exact sequence

$$
0 \longrightarrow \mathcal{O}_{\mathbb{P}_{k}^{2}} \stackrel{A}{\longrightarrow} \mathcal{O}_{\mathbb{P}_{k}^{2}}(2)^{\oplus 6} \longrightarrow \mathcal{E} \longrightarrow 0,
$$

where $\mathcal{E}$ is a locally free $\mathcal{O}_{\mathbb{P}_{k}^{2}}$-sheaf of rank 5 . If $\mathcal{A}_{1}=\mathcal{A}_{2}:=\mathcal{O}_{\mathbb{P}_{k}^{2}}^{\oplus 2}, \mathcal{A}_{3}:=$ $\mathcal{O}_{\mathbb{P}_{k}^{2}}(2)^{\oplus 2}$, then $\check{\mathcal{A}} \otimes \mathcal{E}$ is globally generated thus its general section defines an Anglo-American cover $\varrho: X \rightarrow \mathbb{P}_{k}^{2}$ with Tschirnhausen module $\check{\mathcal{E}}$ : by Proposition 4.1 of [5] we have $q(X)=1, p_{g}(X)=0$.

We claim that such a $\varrho$ cannot decomposes as $\sigma \circ \tau$ as stated in Proposition 4.5 of [6]. If this is the case, then we get decompositions $\tau_{*} \mathcal{O}_{X} \cong \mathcal{O}_{S} \oplus \mathcal{T}$, $\sigma_{*} \mathcal{O}_{S} \cong \mathcal{O}_{\mathbb{P}_{k}^{2}} \oplus \mathcal{S}$ whence $\varrho_{*} \mathcal{O}_{X} \cong \mathcal{O}_{\mathbb{P}_{k}^{2}} \oplus \mathcal{S} \oplus \sigma_{*} \mathcal{T}$. Since $h^{0}\left(\mathbb{P}_{k}^{2}, \check{\mathcal{E}}\right)=0$ the isomorphism $\mathcal{O}_{\mathbb{P}_{k}^{2}} \oplus \mathcal{S} \oplus \sigma_{*} \mathcal{T} \cong \mathcal{O}_{\mathbb{P}_{k}^{2}} \oplus \check{\mathcal{E}}$ induces an isomorphism $\mathcal{S} \oplus \sigma_{*} \mathcal{T} \cong \check{\mathcal{E}}$. Since $\tau$ and $\sigma$ have ranks 2 and 3 respectively, we conclude that $\sigma_{*} \mathcal{T}$ and $\mathcal{S}$ are locally free sheaves of ranks 3 and 2 respectively. Thus it would follow that $h^{0}\left(\mathbb{P}_{k}^{2}, \mathcal{E} \otimes \check{\mathcal{E}}\right) \geq 2$. We now prove that $\mathcal{E}$ is simple, a contradiction.

To this purpose, we observe that the cohomology of the sequence (4.1.1) tensorized by $\check{\mathcal{E}}$ yields the following exact sequence

$$
H^{0}\left(\mathbb{P}_{k}^{2}, \check{\mathcal{E}}(2)\right)^{\oplus 6} \longrightarrow H^{0}\left(\mathbb{P}_{k}^{2}, \mathcal{E} \otimes \check{\mathcal{E}}\right) \longrightarrow H^{1}\left(\mathbb{P}_{k}^{2}, \check{\mathcal{E}}\right) \longrightarrow H^{1}\left(\mathbb{P}_{k}^{2}, \check{\mathcal{E}}(2)\right)^{\oplus 6}
$$

Moreover, taking the cohomology of the dual of (4.1.1) tensorized by $\mathcal{O}_{\mathbb{P}_{k}^{2}}(m)$ we get the following exact sequence

$$
\begin{aligned}
0 \longrightarrow H^{0}\left(\mathbb{P}_{k}^{2}, \check{\mathcal{E}}(m)\right) & \longrightarrow H^{0}\left(\mathbb{P}_{k}^{2}, \mathcal{O}_{\mathbb{P}_{k}^{2}}(m-2)\right) \stackrel{\oplus 6}{{ }^{t} A} \\
& \longrightarrow H^{0}\left(\mathbb{P}_{k}^{2}, \mathcal{O}_{\mathbb{P}_{k}^{2}}(m)\right) \longrightarrow H^{1}\left(\mathbb{P}_{k}^{2}, \check{\mathcal{E}}(m)\right) \longrightarrow 0
\end{aligned}
$$

Trivially $H^{1}\left(\mathbb{P}_{k}^{2}, \check{\mathcal{E}}\right)=1$ (take $m=0$ ). Taking into account the definition of $A$, we get $H^{0}\left(\mathbb{P}_{k}^{2}, \check{\mathcal{E}}(2)\right)=H^{1}\left(\mathbb{P}_{k}^{2}, \check{\mathcal{E}}(2)\right)=0$ (take $m=2$ ). We conclude that $H^{0}\left(\mathbb{P}_{k}^{2}, \mathcal{E} \otimes \check{\mathcal{E}}\right)=H^{1}\left(\mathbb{P}_{k}^{2}, \check{\mathcal{E}}\right)=1$ : thus $\mathcal{E}$ is indecomposable.

Then we give two other examples of covers of degree $d=6$. Recall that (see Theorem 3.1 and Remark 4.2 of [5]) in order to build a Scandinavian cover we need three locally free $\mathcal{O}_{\mathbb{P}_{k}^{2}}$-sheaves, say $\mathcal{E}$ (the Tschirnhausen module of $\varrho$ ), $\mathcal{P}$, $\mathcal{Q}$ of respective ranks $5,3,3$ such that $2 c_{1}(\mathcal{P})-2 c_{1}(\mathcal{Q})=c_{1}(\mathcal{E})$ and such that $\check{\mathcal{P}} \otimes \mathcal{Q} \otimes \mathcal{E}$ is globally generated.

Example 4.2. Let $\mathcal{A}_{1}=\mathcal{A}_{2}=\mathcal{O}_{\mathbb{P}_{k}^{2}}^{\oplus 2}, \mathcal{A}_{3}:=\mathcal{O}_{\mathbb{P}_{k}^{2}}(2) \oplus \mathcal{O}_{\mathbb{P}_{k}^{2}}(3)$ and $\mathcal{E}:=$ $\mathcal{O}_{\mathbb{P}_{k}^{2}}(3)^{\oplus 5}$. Then the general section of $\breve{\mathcal{A}} \otimes \mathcal{E}$ defines an Anglo-American cover $\varrho: X \rightarrow \mathbb{P}_{k}^{2}$ with Tschirnhausen module $\check{\mathcal{E}}$. The cover $\varrho$ cannot be Scandinavian since $c_{1}(\mathcal{E})$ is not even. 
The invariants of $X$ are $q(X)=0, p_{g}(X)=5, K_{X}^{2}=8$ (see Proposition 4.1 of [4]). Twisting sequence (4.1.2) of [4] by $\mathcal{O}_{\mathbb{P}_{k}^{2}}(-6)$ we also get $P_{2}(X)=14$.

Since the natural map $\varrho^{*} \mathcal{E}(-3) \rightarrow \omega_{X}$ is surjective and $\mathcal{E}(-3)$ is globally generated, it follows that the same is true for $\omega_{X}$, thus $X$ is a surface of general type. Moreover, the equality $P_{2}(X)=K_{X}^{2}+\chi\left(\mathcal{O}_{X}\right)$ yields the minimality of $X$.

Example 4.3. Take $\mathcal{E}:=\mathcal{O}_{\mathbb{P}_{k}^{2}}(2)^{\oplus 5}, \mathcal{P}:=\mathcal{O}_{\mathbb{P}_{k}^{2}}^{\oplus 3}, \mathcal{Q}:=\mathcal{O}_{\mathbb{P}_{k}^{2}}(-1) \oplus \mathcal{O}_{\mathbb{P}_{k}^{2}}(-2)^{\oplus 2}$. One check that the above conditions are satisfied, so we obtain a Scandinavian cover $\varrho: X \rightarrow \mathbb{P}_{k}^{2}$ which cannot be Anglo-American since $c_{1}(\mathcal{E})$ is not a multiple of three in $\operatorname{Pic}\left(\mathbb{P}_{k}^{2}\right)$ (see Remark 2.8). By Proposition 4.1 of [5] we have $X$ are $q(X)=p_{g}(X)=0$.

One could ask if each Gorenstein cover of degree 6 is either Scandinavian or Anglo-American: the answer to this question is negative as the following example shows.

Example 4.4. Consider in $\mathbb{P}:=\mathbb{P}\left(\mathcal{O}_{\mathbb{P}_{k}^{1}}(2)^{\oplus 3}\right) \stackrel{\pi}{\longrightarrow} \mathbb{P}_{k}^{1}$ two general divisors $F \in$ $\left|\mathcal{O}_{\mathbb{P}}(2)\right|$ and $G \in\left|\mathcal{O}_{\mathbb{P}}(3) \otimes \pi^{*} \mathcal{O}_{\mathbb{P}_{k}^{1}}(1)\right|$. Then it is easy to check that $X:=F \cap G$ is a smooth curve, thus $\varrho:=\pi_{\mid X}: X \rightarrow \mathbb{P}_{k}^{1}$ is a Gorenstein cover which turns to have degree 6 by construction.

Tensoring

$$
0 \longrightarrow \mathcal{O}_{\mathbb{P}}(-3) \otimes \pi^{*} \mathcal{O}_{\mathbb{P}_{k}^{1}}(-1) \longrightarrow \mathcal{O}_{\mathbb{P}} \longrightarrow \mathcal{O}_{G} \longrightarrow 0
$$

by $\mathcal{O}_{\mathbb{P}}(2) \otimes \pi^{*} \mathcal{O}_{\mathbb{P}_{k}^{1}}(7) \otimes \mathcal{O}_{F}$, pushing down, taking into account that $\omega_{X \mid \mathbb{P}_{k}^{1}} \cong$ $\mathcal{O}_{\mathbb{P}}(2) \otimes \pi^{*} \mathcal{O}_{\mathbb{P}_{k}^{1}}(7) \otimes \mathcal{O}_{X}$ and relative duality we obtain the sequence

$$
\begin{aligned}
0 \longrightarrow \pi_{*}\left(\mathcal{O}_{\mathbb{P}}(2) \otimes \pi^{*} \mathcal{O}_{\mathbb{P}_{k}^{1}}(7) \otimes \mathcal{O}_{F}\right) \longrightarrow \varrho_{*} \omega_{X \mid \mathbb{P}_{k}^{1}} \\
\longrightarrow R^{1} \pi_{*}\left(\mathcal{O}_{\mathbb{P}}(-1) \otimes \pi^{*} \mathcal{O}_{\mathbb{P}_{k}^{1}}(6) \otimes \mathcal{O}_{F}\right) \longrightarrow 0 .
\end{aligned}
$$

Now sequence

$$
0 \longrightarrow \mathcal{O}_{\mathbb{P}}(-2) \longrightarrow \mathcal{O}_{\mathbb{P}} \longrightarrow \mathcal{O}_{F} \longrightarrow 0
$$

and relative duality yields $R^{1} \pi_{*}\left(\mathcal{O}_{\mathbb{P}}(-1) \otimes \pi^{*} \mathcal{O}_{\mathbb{P}_{k}^{1}}(6) \otimes \mathcal{O}_{F}\right) \cong \mathcal{O}_{\mathbb{P}_{k}^{1}}$, thus $\mathcal{E} \cong$ $\pi_{*}\left(\mathcal{O}_{\mathbb{P}}(2) \otimes \pi^{*} \mathcal{O}_{\mathbb{P}_{k}^{1}}(7) \otimes \mathcal{O}_{F}\right)$. Again sequence (4.4.1) twisted accordingly yields $\mathcal{E} \cong \operatorname{coker}\left(\mathcal{O}_{\mathbb{P}_{k}^{1}} \rightarrow \pi_{*} \mathcal{O}_{\mathbb{P}}(2)\right) \otimes \pi^{*} \mathcal{O}_{\mathbb{P}_{k}^{1}}(7)$, hence a Chern classes computation gives us $c_{1}(\mathcal{E})^{k}=59$ which is neither even nor a multiple of 3 . In particular, such a cover $\varrho$ is neither Scandinavian nor Anglo-American.

\section{5. $(1,3)$-polarized abelian surfaces}

Let $\mathcal{A}_{1}=\mathcal{A}_{2}=\mathcal{A}_{3}:=\Omega_{\mathbb{P}_{k}^{2}}^{1}(2)$ be the sheaf of differentials on $\mathbb{P}_{k}^{2}$ and define $\mathcal{E}:=\mathcal{O}_{\mathbb{P}_{k}^{2}}(3) \oplus \Omega_{\mathbb{P}_{k}^{2}}^{1}(3) \oplus \Omega_{\mathbb{P}_{k}^{2}}^{1}(3)$. We ask if there are sections $\eta$ of $\check{\mathcal{A}} \otimes \mathcal{E}$ defining Gorenstein covers $\varrho: A \rightarrow \mathbb{P}_{k}^{2}$. Though we cannot make use of any Bertini theorem since $\breve{\mathcal{A}} \otimes \mathcal{E}$ is unfortunately not globally generated, we have the following: 
Lemma 5.1. $\Omega_{\mathbb{P}_{k}^{2}}^{1} \otimes \Omega_{\mathbb{P}_{k}^{2}}^{1} \otimes \Omega_{\mathbb{P}_{k}^{2}}^{1} \cong \mathcal{S}^{3} \Omega_{\mathbb{P}_{k}^{2}}^{1} \oplus \Omega_{\mathbb{P}_{k}^{2}}^{1}(-3) \oplus \Omega_{\mathbb{P}_{k}^{2}}^{1}(-3)$.

Proof. We have the decomposition $\Omega_{\mathbb{P}_{k}^{2}}^{1} \otimes \Omega_{\mathbb{P}_{k}^{2}}^{1} \cong \mathcal{S}^{2} \Omega_{\mathbb{P}_{k}^{2}}^{1} \oplus \mathcal{O}_{\mathbb{P}_{k}^{2}}(-3)$. The statement then follows by tensoring by $\Omega_{\mathbb{P}_{k}^{2}}^{1}$ and applying Formula (6.7) of [9] (globalizing Gordan-Clebsch formula).

It follows that $\eta$ can be identified with a matrix

$$
\left(\begin{array}{lll}
\eta_{1,1} & \eta_{1,2} & \eta_{1,3} \\
\eta_{2,1} & \eta_{2,2} & \eta_{2,3} \\
\eta_{3,1} & \eta_{3,2} & \eta_{3,3}
\end{array}\right)
$$

where $\eta_{1,1} \in H^{0}\left(\mathbb{P}_{k}^{2}, \mathcal{S}^{3} \Omega_{\mathbb{P}_{k}^{2}}^{1}(6)\right), \eta_{1,2}, \eta_{1,3} \in H^{0}\left(\mathbb{P}_{k}^{2}, \Omega_{\mathbb{P}_{k}^{2}}^{1}(3)\right), \eta_{2,1}, \eta_{3,1} \in$ $H^{0}\left(\mathbb{P}_{k}^{2}, \Omega_{\mathbb{P}_{k}^{2}}^{1} \otimes \mathcal{S}^{3} \Omega_{\mathbb{P}_{k}^{2}}^{1}(6)\right)$ and $\eta_{2,2}, \eta_{2,3}, \eta_{3,2}, \eta_{3,3} \in H^{0}\left(\mathbb{P}_{k}^{2}, \Omega_{\mathbb{P}_{k}^{2}}^{1} \otimes \Omega_{\mathbb{P}_{k}^{2}}^{1}(3)\right)$. Since $\mathcal{S}^{3} \Omega_{\mathbb{P}_{k}^{2}}^{1}(6)$ is globally generated and $\Omega_{\mathbb{P}_{k}^{2}}^{1}$ is simple, whence $H^{0}\left(\mathbb{P}_{k}^{2}, \Omega_{\mathbb{P}_{k}^{2}}^{1} \otimes \Omega_{\mathbb{P}_{k}^{2}}^{1}(3)\right)$ $\cong H^{0}\left(\mathbb{P}_{k}^{2}, \Omega_{\mathbb{P}_{k}^{2}}^{1} \otimes \Omega_{\mathbb{P}_{k}^{2}}^{1}\right) \cong k$, the following lemma follows trivially.

Lemma 5.2. The locus

$$
H:=\left\{\eta \in H^{0}\left(\mathbb{P}_{k}^{2}, \check{\mathcal{A}} \otimes \mathcal{E}\right) \mid \eta: \Omega_{\mathbb{P}_{k}^{2}}^{1} \otimes \Omega_{\mathbb{P}_{k}^{2}}^{1} \otimes \Omega_{\mathbb{P}_{k}^{2}}^{1}(6) \rightarrow \mathcal{E} \text { is surjective }\right\}
$$

is non-empty and open.

Thus each general $\eta \in H^{0}\left(\mathbb{P}_{k}^{2}, \check{\mathcal{A}} \otimes \mathcal{E}\right)$ defines an embedding $\mathbb{P}=\mathbb{P}(\mathcal{E}) \subseteq \overline{\mathbb{P}}=$ $\mathbb{P}\left(\Omega_{\mathbb{P}_{k}^{2}}^{1} \otimes \Omega_{\mathbb{P}_{k}^{2}}^{1} \otimes \Omega_{\mathbb{P}_{k}^{2}}^{1}(6)\right)$, whence we can consider the scheme $A:=D_{t r}(\Phi(\eta))=$ $\mathcal{D}_{t r} \cap \mathbb{P} \subseteq \mathbb{P}$ and the morphism $\varrho:=\pi_{\mid A}: A \rightarrow \mathbb{P}_{k}^{2}$.

Assume that $\eta$ has the right codimension at each $y \in \mathbb{P}_{k}^{2}:$ then $\varrho: A \rightarrow \mathbb{P}_{k}^{2}$ is a Gorenstein cover. Moreover, we have the following:

Proposition 5.3. Let $\eta$ be as above. If $A$ is smooth, then it is an abelian surface with a natural polarization of type $(1,3)$ induced by $\varrho$.

Proof. By Proposition 4.1 of [5] we have $h^{0}\left(A, \mathcal{O}_{A}\right)=1$, thus $A$ is connected, hence, being smooth, it is also integral. Moreover, $q(A)=2$ and $p_{g}=1$, thus there are effective canonical divisors.

For each cover $\varrho$, its branch locus $B$ is in $\left|\operatorname{det} E^{2}\right|$ (see [1], Proposition 6.6). Thus, in our setup, $\operatorname{deg}(B)=18$, then for each general line $r \subseteq \mathbb{P}_{k}^{2}$, the curve $C:=\varrho^{-1}(r)$ is smooth and connected of genus $g=4$.

If $K_{A}$ is a canonical divisor on $A$, then $6=2 g-2=K_{A} \cdot C+C^{2}=k_{A} \cdot C+6$ by adjunction on $A$, thus $K_{A} \cdot C=0$. Since $C$ is ample as divisor on $A$, then $K_{A}=0$, i.e., $A$ is abelian and $\varrho^{*} \mathcal{O}_{\mathbb{P}_{k}^{2}}(1)$ is a polarization on $A$ of type $(1,3)$.

It is then natural to ask the following.

Question 5.4. Does the aforementioned genericity assumption actually hold for some sections $\eta$ ? If this is the case, are all the $(1,3)$-polarized abelian surfaces obtained via the above construction? 


\section{References}

[1] A. Altman and S. Kleiman, Introduction to Grothendieck Duality Theory, Lecture Notes in Mathematics, Vol. 146 Springer-Verlag, Berlin-New York, 1970.

[2] E. Arbarello, M. Cornalba, P. A. Griffiths, and J. Harris, Geometry of Algebraic Curves. Vol. I, Springer-Verlag, New York, 1985.

[3] G. Casnati, Covers of algebraic varieties II. Covers of degree 5 and construction of surfaces, J. Algebraic Geom. 5 (1996), no. 3, 461-477.

[4] - The cover associated to a (1,3)-polarized bielliptic abelian surface and its branch locus, Proc. Edinburgh Math. Soc. (2) 42 (1999), no. 2, 375-392.

[5] _ Covers of algebraic varieties IV. A Bertini theorem for Scandinavian covers, Forum Math. 13 (2001), no. 1, 21-36.

6] _ Covers of algebraic varieties $V$. Examples of covers of degree 8 and 9 as catalecticant loci, J. Pure Appl. Algebra 182 (2003), no. 1, 17-32.

[7] G. Casnati and T. Ekedahl, Covers of algebraic varieties I. A general structure theorem, covers of degree 3, 4 and Enriques surfaces, J. Algebraic Geom. 5 (1996), no. 3, 439-460.

[8] G. Casnati and R. Notari, On some Gorenstein loci in $\mathcal{H}$ ilb $6\left(\mathbb{P}_{k}^{4}\right)$, J. Algebra 308 (2007), no. 2, 493-523.

[9] W. Fulton and J. Harris, Representation Theory: A First Course, Graduate Texts in Mathematics, 129, Springer-Verlag, New York, 1991

[10] Ph. Griffiths and J. Harris, Principles of Algebraic Geometry, John Wiley \& Sons, 1978.

[11] A. Grothendieck, Techniques de construction et théorèmes d'existence en géométrie algébrique, IV: les schémas de Hilbert, Extraits du séminaire Bourbaki, Sécretariat Mathématique, 1961.

[12] R. Hartshorne, Algebraic Geometry, Graduate Texts in Mathematics, No. 52.SpringerVerlag, New York-Heidelberg, 1977.

[13] V. A. Iskovskikh and Yu. G. Prokhorov, Fano Varieties, Algebraic Geometry V, A. N. Parshin and I. R. Shafarevich, Encyclopedia of Mathematical Sciences, Vol. 47, 1991.

[14] A. R. Kustin and M. Miller, Structure theory for a class of grade four Gorenstein ideals, Trans. Amer. Math. Soc. 270 (1982), no. 1, 287-307.

[15] H. Lange and E. Sernesi, Severi varieties and branch curves of abelian surfaces of type (1,3), Internat. J. Math. 13 (2002), no. 3, 227-244.

[16] A. Lascoux, Syzygies des variétés déterminantales, Adv. in Math. 30 (1978), no. 3, $202-237$.

[17] J. C. Migliore, Introduction to Liaison Theory and Deficiency Modules, Progress in Mathematics, Vol. 165, Birkhäuser, 1998.

[18] R. Miranda, Triple covers in algebraic geometry, Amer. J. Math. 107 (1985), no. 5 $1123-1158$.

[19] D. Mumford, Lectures on Curves on an Algebraic Surface, Princeton University Press, Princeton, N.J. 1966.

[20] F. O. Schreyer, Syzygies of canonical curves and special linear series, Math. Ann. 275 (1986), no. 1, 105-137.

Dipartimento di Matematica

Politecnico di Torino

C.SO DuCA Degli Abruzzi 24

10129 Torino, ItALY

E-mail address: casnati@calvino.polito.it 